\title{
Efficacy and safety of glucosamine, diacerein, and NSAIDs in osteoarthritis knee: a systematic review and network meta-analysis
}

\author{
Jatupon Kongtharvonskul ${ }^{1 *}$, Thunyarat Anothaisintawee ${ }^{1}$, Mark McEvoy ${ }^{2}$, John Attia ${ }^{3}$, Patarawan Woratanarat $^{4}$ \\ and Ammarin Thakkinstian ${ }^{1}$
}

\begin{abstract}
Background: To conduct a systematic review and network meta-analysis of randomized controlled trials (RCTs) with the aims of comparing relevant clinical outcomes (that is, visual analog scores (VAS), total and sub-Western Ontario and McMaster Universities Osteoarthritis index (WOMAC) scores, Lequesne algofunctional index, joint space width change, and adverse events) between diacerein, glucosamine, and placebo.

Methods: Medline and Scopus databases were searched from inception to 29 August 2014, using PubMed and Scopus search engines and included RCTs or quasi-experimental designs comparing clinical outcomes between treatments. Data were extracted from original studies. A network meta-analysis was performed by applying weight regression for continuous outcomes and a mixed-effect Poisson regression for dichotomous outcomes.

Results: Thirty-one of 505 identified studies were eligible. Compared to placebo, glucosamine showed a significant improvement with unstandardized mean differences (UMD) in total WOMAC, pain WOMAC, function WOMAC, and Lequesne score of -2.49 (95\% confidence interval $(\mathrm{Cl})-4.14,-0.83),-0.75(95 \% \mathrm{Cl}:-1.18,-0.32),-4.78$ (95\% Cl: $-5.96,-3.59)$, and -1.03 (95\% Cl: $-1.34,-0.72)$, respectively. Diacerein clinically improves visual analog scores, function WOMAC, and stiffness WOMAC with UMD values of -2.23 (95\% Cl: $-2.82,-1.64),-6.64$ (95\% $\mathrm{Cl}:-10.50,-2.78)$, and $-0.68(95 \% \mathrm{Cl}:-1.20,-0.16)$ when compared to placebo.
\end{abstract}

Conclusions: The network meta-analysis suggests that diacerein and glucosamine are equally efficacious for symptom relief in knee OA, but that the former has more side effects.

Keywords: Osteoarthritis, Gonarthrosis, Systematic review, Network meta-analysis, SYSADOA

\section{Background}

Osteoarthritis (OA) is the most common chronic joint disease of the older patient. The primarily affected joints are the knee and hip. The progression of the disease is influential on quality of life. This included functional and social activities, body image, and emotional well-being. In non-operative treatment, pain reduction and improved function are the primary goals. Management of mild degree OA of the knee mainly consists of medical treatment and lifestyle modifications. Non-steroidal anti-

\footnotetext{
* Correspondence: Jatupon_kong@hotmail.com

'Section for Clinical Epidemiology and Biostatistics, Faculty of Medicine, Faculty of Medicine Ramathibodi Hospital, Ratchathewi, Chang Wat Bangkok, Bangkok 10400, Thailand

Full list of author information is available at the end of the article
}

inflammatory drugs (NSAIDs) are the most commonly prescribed agents for pain management, but they increase the risk of gastrointestinal (GI) bleeding and vascular adverse events $[1,2]$. Therefore, second-line drugs such as symptomatic slow-acting drugs for OA (SYSADOA) which include glucosamine sulfate, glucosamine hydrochloride, chondroitin sulfate, hyaluronic acid, avocado soybean unsaponifiables (ASU), and diacerein are more commonly used. These drugs may improve patient symptoms as well as reduce cartilage degradation $[3,4]$, also having decreased occurrence of GI adverse events when compared to NSAIDs. Two drugs are recommended by the European League Against Rheumatism guidelines 2003. These include an interleukin-1 (IL-1B) inhibitor (diacerein) and glucosamine. However, these drugs 
have a slow onset and a prolonged residual effect. The diacerein and glucosamine groups have the greatest amount of randomized controlled trial (RCT) studies and meta-analysis when compared to all other SYSADOA. The results of all the studies show that diacerein and glucosamine improve symptoms and decrease structural progression in OA of the knee when compared to NSAIDs and placebo. Previous systematic reviews [3-6] have shown that diacerein had higher efficacy in reducing pain and Lequesne index, but increased risk of diarrhea when compared to placebo $[4,6]$. Similar effects were observed in systematic reviews of the efficacy of glucosamine, which showed a significant reduction in pain when compared to placebo but no effect on minimal joint space narrowing or adverse events [3,5]. However, no RCTs directly compared the clinical efficacy and safety of diacerein with glucosamine. We therefore conducted a systematic review with a network meta-analysis of RCTs with the aim of comparing relevant clinical outcomes (that is, visual analog score, Western Ontario and McMaster Universities Osteoarthritis index (WOMAC) score, Lequesne algofunctional index, joint space width change, and adverse events) between diacerein, glucosamine, NSAIDs, and placebo. The main outcomes that were focused on in this study included pain, functional assessment, joint space width change, and safety issues of the medications.

\section{Methods}

\section{Search strategy}

The Medline and Scopus databases were used to identify relevant studies published in English from the date of inception to 29 August 2014. The PubMed and Scopus search engines were used to locate studies using the following search terms: (osteoarthritis, degenerative arthritis, adult, older person), (symptomatic slow acting drug for osteoarthritis; SYSADOA, diacerein, glucosamine), (pain, function, score, grade, WOMAC, Knee Society Score (KSS), motion, radiographic grading, X-ray, MRI, Kellgren-Lawrence), (clinical trial, RCT, randomized controlled trial). Search strategies for Medline and Scopus are described in Additional file 1. Relevant studies from the reference lists of identified studies and previous systematic reviews were also explored.

\section{Selection of studies}

Identified studies were selected by one author (J.K.) and randomly checked by A.T. Titles and abstracts were initially screened; full papers were then retrieved if a decision could not be made from the abstracts. The reasons for ineligibility or exclusion of studies were recorded and described (Figure 1).

\section{Inclusion criteria}

Randomized controlled trials or quasi-experimental designs comparing clinical outcomes between treatments in primary OA patients' knee were eligible if they met the following criteria:

- Compared clinical outcomes between glucosamine (either glucosamine sulfate or glucosamine hydrochloride) and diacerein, or each of these treatments with other comparators (for example, placebo, non-steroidal anti-inflammatory drugs).

- Compared at least one of the following outcomes: pain score, function, patient/physician global assessments, range of motion, joint space width difference, and adverse events.

- Had sufficient data to extract and pool: reported mean, standard deviation (SD), numbers of subjects according to treatments for continuous outcomes, and number of patients according to treatment for dichotomous outcomes.

\section{Data extraction}

Two reviewers (J.K. and T.A.) independently performed data extraction using standardized data extraction forms. General characteristics of the study (mean age, gender, body mass index, duration of OA, pain score, and functional scores at baseline) were extracted. The number of subjects, mean, and SD of continuous outcomes (pain by visual analog score (VAS), total and sub-WOMAC scores, and Lequesne algofunctional index) between the groups were extracted. Cross-tabulated frequencies between treatments and adverse events were also extracted. Any disagreements were resolved by discussion and consensus with a third party (A.T.).

\section{Risk of bias assessment}

Two authors (J.K. and T.A.) independently assessed risk of bias for each study. Six study quality domains were considered, including sequence generation, allocation concealment, blinding (participant, personnel, and outcome assessors), incomplete outcome data, selective outcome reporting, and other sources of bias [7]. Disagreements between two authors were resolved by consensus and discussion with a third party (A.T.).

\section{Outcomes}

The outcome of interests were pain VAS, total and subWOMAC scores (pain, stiffness, and function), Lequesne algofunctional index, joint space width (minimum), and adverse events. Methods of measure for these outcomes were used according to the original studies. Briefly, this includes the VAS pain scale from 0 to 10 ; the WOMAC score that consists of pain (0 to 20), stiffness (0 to 8), and function (0 to 68) with total scores of 0 to 96 [8]. 


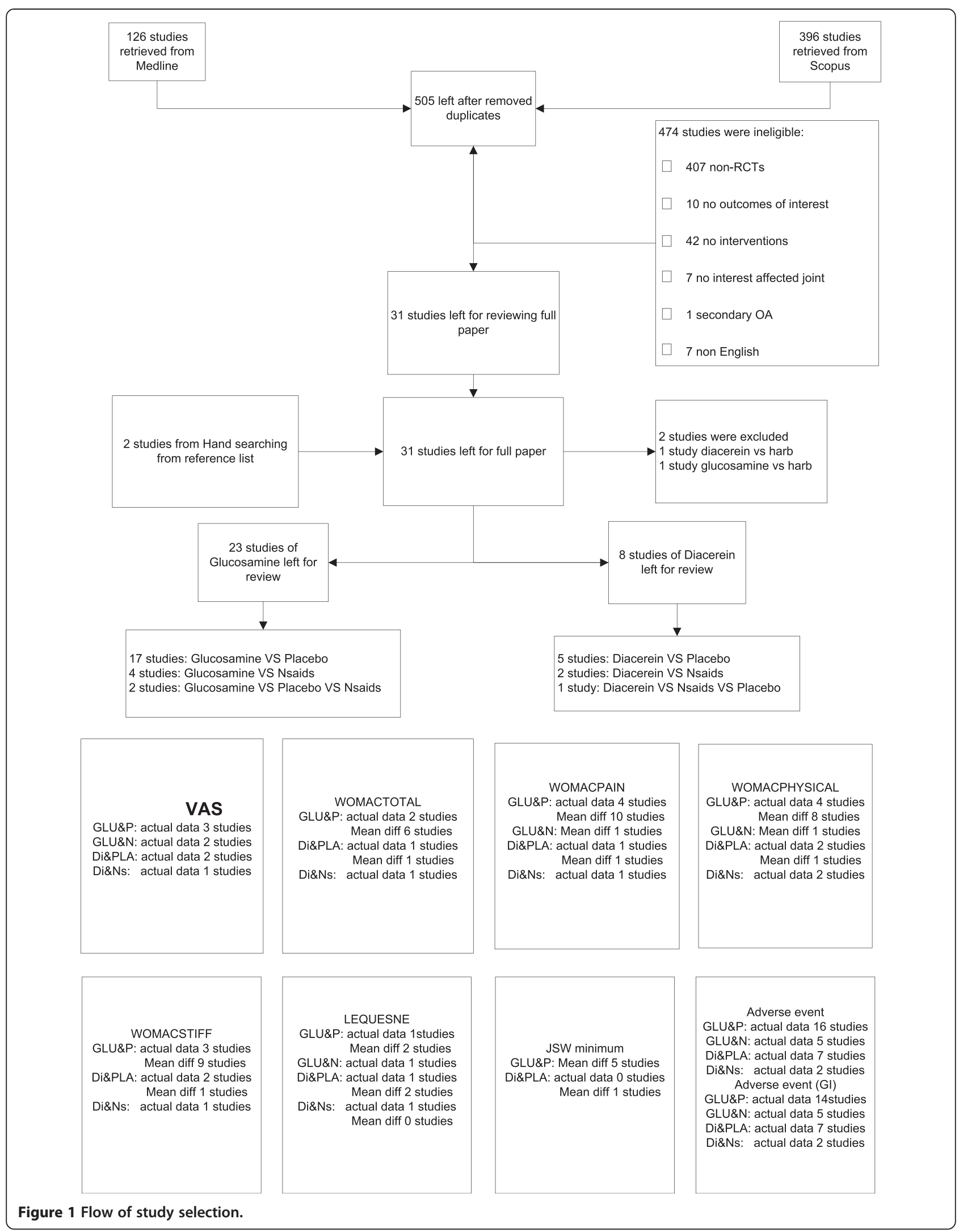


The Lequesne algofunctional index measured pain (0 to 10), maximum distance walked (0 to 6), and activities of daily living (0 to 8$)$ with total scores of 0 to 24 [9,8]. For joint space width change, lower values of these scores refer to better outcomes. Adverse events were considered as composite and separate outcomes of the following: a musculoskeletal disorder, respiratory disorder, genitourinary tract disorder or central nervous system disorder, and GI adverse effects.

\section{Statistical analysis}

Direct comparisons of continuous outcomes were measured at the end of each study between glucosamine versus placebo and diacerein versus placebo and were then pooled using an unstandardized mean difference (UMD). Heterogeneity of the mean difference across studies was checked using the $Q$ statistic, and the degree was quantified using the $I^{2}$ statistic. If heterogeneity was present $(P$ value $<0.10$ or the $\left.I^{2}>25 \%\right)$, the UMD was estimated using a random effects model; otherwise, a fixed-effects model was applied.

For dichotomous outcomes, a relative risk (RR) of adverse reactions of treatment comparisons at the end of each study was estimated and pooled. Heterogeneity was assessed using the same method as mentioned previously. If heterogeneity was present, the DerSimonian and Laird method [10] was applied for pooling; otherwise, the fixedeffects model by inverse variance method was applied. Meta-regression was applied to explore the source of heterogeneity (for example, mean age, percentage of females, bone mass index (BMI), Kellgren-Lawrence grading, duration of $\mathrm{OA}$ ) if data was available. Publication bias was assessed using contour-enhanced funnel plots [11,12] and Egger tests [13].

For indirect comparisons, network meta-analyses were applied to assess all possible effects of treatment measured at different times if summary data were available for pooling [14-16]. A linear regression model weighted by inverse variance was applied to assess the treatment effects with adjustment for study effects and time for continuous outcomes. For adverse events, a mixed-effect Poisson regression was applied to assess treatment effects [15]. Summary data was expanded to individual patient data using the "expand" command in STATA. Treatment was considered as a fixed-effect, whereas the study variable was considered as a random-effect in a mixed-effect model. The pooled RR and its 95\% confidence intervals (CIs) were estimated by exponential coefficients of treatments. All analyses were performed using STATA version 12.0 [17]. A $P$ value $<0.05$ was considered statistically significant, except for the test of heterogeneity where $P$ value $<0.10$ was used.

\section{Results}

Among 505 identified studies and 2 referred studies, 31 studies [18-48] were eligible for data extraction. Reasons for ineligibility are described in Figure 1. Characteristics of the 31 studies [18-44,46-48,45] are described in Table 1.

Among 23 glucosamine studies [26-44,1-4], the comparators included placebo, NSAIDs, and both placebo and NSAIDS in 17 studies [39,30,29,33,43,31,42,41,32 ,38,26,27,35,37,4,1,2], 4 studies [40,36,34,48,28], and 2 studies $[28,44]$, respectively. All studies used glucosamine sulfate, except for one study [31] which used glucosamine hydrochloride. Among eight diacerein studies, five studies $[21,23,19,22,18]$, two studies [25,20], and 1 study [21] had comparators as placebo, NSAIDs, and both NSAIDs and placebo, respectively. Most studies (24/27) included OA of the knee and the rest were OA of the hip. Mean age, body mass index (BMI), and duration of OA varied from 42 to 69 years, 24.0 to $32.6 \mathrm{~kg} / \mathrm{m}^{2}$, and 1.6 to 13 years, respectively. Percentage of females in each study ranged from $5.1 \%$ to $88 \%$. Duration of treatment ranged from 4 weeks to 3 years. Various outcomes were compared between the treatment groups (Figure 1).

\section{Risk of bias in included studies}

Risk of bias assessment is described in Additional file 2.

\section{Direct comparisons}

Data used for direct comparisons for all treatments and outcomes were measured at the end of each study, as described in Table 1. Pooling according to outcomes was performed if there were at least two studies for each comparison, as clearly described below.

\section{Visual analog score}

Among eight studies [25,39,34,21,23,40,42,47], five studies compared glucosamine pain VAS with comparators of placebo $[39,42,2]$ and NSAIDs [34,40], respectively. Three studies compared diacerein with placebo $[21,23]$ and NSAIDs [25]. Most studies assessed pain using the VAS at 4 weeks to 3 years. Data for the mean and SD of VAS scores are described in Additional file 3. The mean VAS was -0.90 (95\% CI: $-1.67,-0.14)$ units significantly lower in glucosamine than in NSAIDs (Table 2). The mean VAS score was about -1.44 (95\% CI: -3.01, 0.12) units lower in glucosamine than in placebo, but this was not significant. The pooled effects of diacerein versus placebo from three studies $(n=103$ vs. 98) displayed no heterogeneity $\left(I^{2}=0 \%\right)$ with an UMD of -2.23 (95\% CI: $-2.82,-1.64$ ). The effect of diacerein versus NSAIDs was not statistically different with an UMD of 0.149 (95\% CI: $-0.29,0.59$ ). There was no evidence of publication bias for both pooled effect estimates. 
Table 1 Characteristics of included studies

\begin{tabular}{|c|c|c|c|c|c|c|c|c|c|}
\hline Author & Years & Follow-up & $\begin{array}{l}\text { Intervention } \\
\text { (mg) }\end{array}$ & Comparator & Age & $\begin{array}{l}\text { Female } \\
(\%)\end{array}$ & BMI & Duration & Outcome \\
\hline Pujalte JM & 1980 & 8 weeks & GS $(1,500)$ & Placebo & 61.7 & 85 & - & - & VAS, AR \\
\hline Lopes VA & 1982 & 8 weeks & GS $(1,500)$ & NSAIDs & 56.4 & 74 & - & 3.2 & VAS, AR \\
\hline Muller FH & 1994 & 4 weeks & GS $(1,500)$ & NSAIDs & 54 & 42.3 & - & 4.8 & Lequesne, AR \\
\hline Noack W & 1994 & 4 weeks & GS $(1,500)$ & Placebo & 55 & 60.3 & - & - & Lequesne, AR \\
\hline Nguyen M & 1994 & 8 weeks & $\mathrm{D}(50)$ & NSAIDs, placebo & 62 & 62.7 & - & 5.25 & VAS, Lequesne, AR \\
\hline Qiu GX & 1998 & 4 weeks & GS $(1,500)$ & NSAIDs & 56.4 & 79 & - & - & VAS, AR \\
\hline Houpt JB & 1999 & 8 weeks & $\mathrm{GH}(1,500)$ & Placebo & 64.5 & 64.4 & - & 8.3 & WOMAC, AR \\
\hline Rindone JP & 2000 & 4,8 weeks & GS $(1,500)$ & Placebo & 63.5 & 5.1 & - & 13 & VAS, AR \\
\hline Pelletier JP & 2000 & 24 weeks & $D(50,100,150)$ & Placebo & 62.8 & 76 & 31.28 & 7.9 & VAS \\
\hline Reginster JY & 2001 & 3 years & GS $(1,500)$ & Placebo & 65.8 & 79.4 & 27.35 & 7.8 & WOMAC, JSW, AR \\
\hline Dougados M & 2001 & 3 years & $D(100)$ & Placebo & 62.6 & 84 & - & - & Lequesne, JSW \\
\hline Hughes R & 2002 & 6 months & GS $(1,500)$ & Placebo & 62.3 & 68 & - & 7.63 & $A R$ \\
\hline Pavelka K & 2002 & $1,2,3$ years & GS $(1,500)$ & Placebo & 62.4 & 78.5 & 25.7 & 10.55 & Lequesne, JSW, AR \\
\hline Braham R & 2003 & 3 months & GS $(2,000)$ & Placebo & 42.2 & 28.3 & - & 12.97 & $A R$ \\
\hline Cibere J & 2004 & 24 weeks & GS $(1,500)$ & Placebo & 64.5 & 57.7 & 25.26 & 1.6 & $A R$ \\
\hline McAlindon T & 2004 & 12 weeks & GS $(1,500)$ & Placebo & - & 64 & 32.57 & - & WOMAC, AR \\
\hline Pham T & 2004 & 1 year & $D(100)$ & Placebo & 64.7 & 65.3 & 29.6 & - & Lequesne \\
\hline Clegg DO & 2006 & 24 weeks & GS $(1,500)$ & Placebo, NSAIDs & 58.7 & 42.2 & 31.73 & 10 & WOMAC, AR \\
\hline Zheng WJ & 2006 & 12,16 weeks & $D(100)$ & NSAIDs & 56.2 & 66.2 & 26.13 & 7.23 & VAS, WOMAC, AR \\
\hline Herrero-Beaumont G & 2007 & 24 weeks & GS $(1,500)$ & Placebo & 63.9 & 88 & 27.65 & 7.31 & Lequesne, AR \\
\hline Louthrenoo W & 2007 & 12,24 weeks & $D(50)$ & NSAIDs & 54 & 73 & 26.85 & 3.58 & WOMAC \\
\hline Pavelka K & 2007 & 12,24 weeks & $D(50)$ & Placebo & 63.7 & 65.5 & 28.9 & 6.497 & WOMAC, AR \\
\hline Frestedt JL & 2008 & 12 weeks & GS $(1,500)$ & Placebo & 59.1 & 60 & 32.24 & - & WOMAC, AR \\
\hline Kawasaki T & 2008 & 18 weeks & GS $(1,500)$ & Placebo & 68.9 & - & 23.95 & - & JSW \\
\hline Rozendaal RM & 2008 & $3,12,24$ months & GS $(1,500)$ & Placebo & 63.4 & 69.4 & 27.95 & - & JSW, AR \\
\hline Sawitzke AD & 2008 & 2 months & GS $(1,500)$ & Placebo, NSAIDs & 50.6 & 55.7 & - & 8.52 & JSW \\
\hline Brahmachari B & 2009 & 12 weeks & $D(50)$ & Placebo & 49.1 & 83.6 & 24.66 & 2.76 & VAS, WOMAC, AR \\
\hline Madhu K & 2013 & 6 weeks & GS $(1,500)$ & Placebo & 56.8 & 70 & 27.9 & - & VAS, AR \\
\hline Chopra A & 2013 & 24 weeks & GS $(1,500)$ & NSAIDs & 55.5 & - & 27.7 & - & WOMAC, AR \\
\hline Durmus D & 2013 & 12 weeks & GS $(1,500)$ & Placebo & 55.8 & - & 27.7 & - & WOMAC \\
\hline Kwoh CK & 2014 & 24 weeks & GS $(1,500)$ & Placebo & 52.24 & 48.9 & 28.9 & - & WOMAC, AR \\
\hline
\end{tabular}

$\mathrm{AR}=$ adverse event, $\mathrm{BMI}=$ body mass index $\mathrm{D}=$ diacerein, $\mathrm{GH}=$ glucosamine hydrochloride, $\mathrm{GS}=$ glucosamine sulfate, JSW = joint space width, $\mathrm{VAS}=$ visual analog score.

\section{WOMAC score}

As described in Table 2, the total WOMAC scores were compared as change from baseline and the actual scores measured at the end of each study. Among six studies $[38,27,35,30,29,33]$ with total WOMAC score changes, the effects displayed no heterogeneity $\left(I^{2}=0 \%\right)$ with an UMD of -2.49 (95\% CI: $-4.14,-0.83)$. The actual total WOMAC scores from two studies $[31,29]$ were not statistically significant with an UMD of 5.67 (95\% CI: -11.26 , 22.61). The actual total WOMAC scores and change in sub-WOMAC scores (pain, stiffness, and function) were also compared (Table 2). Compared with placebo, glucosamine resulted in a significantly greater change in WOMAC pain scores with an UMD of $-0.75(95 \%$ $\mathrm{CI}:-1.18,-0.32)$. In addition, mean functional and stiffness WOMAC scores were significantly lower in the diacerein groups when compared to the NSAIDs and placebo groups (6.64 (95\% CI: -10.50, -2.78) and -0.68 (95\% CI: $-1.20,-0.16)$ ).

\section{Lequesne score and joint space width}

Only glucosamine resulted in a significantly greater change of Lequesne score when compared to placebo $(\mathrm{UMD}=-1.030 \quad(95 \%$ CI: $-1.34,-0.72)) \quad($ Table 2$)$. 
Table 2 Summarized results of direct comparisons according to type of interventions

\begin{tabular}{|c|c|c|c|c|}
\hline Clinical outcomes & No. studies & 12 & No. subjects & UMD (95\% Cl) \\
\hline \multicolumn{5}{|l|}{ VAS } \\
\hline Glu vs. Pla & 3 & 83.4 & 89 vs. 89 & $-1.44(-3.01,0.12)$ \\
\hline Glu vs. NSAIDs & 2 & 0 & 106 vs. 110 & $-0.90(-1.67,-0.14)^{*}$ \\
\hline Dia vs. Pla & 2 & 0 & 103 vs. 98 & $-2.23(-2.82,-1.64)^{*}$ \\
\hline Dia vs. NSAIDs & 2 & 0 & 181 vs. 182 & $0.15(-0.29,0.59)$ \\
\hline \multicolumn{5}{|c|}{ Total WOMAC score change } \\
\hline Glu vs. Pla & 6 & 0 & 437 vs. 423 & $-2.49(-4.14,-0.83)^{*}$ \\
\hline \multicolumn{5}{|l|}{ Actual score } \\
\hline Glu vs. Pla & 2 & 79.8 & 73 vs. 76 & $5.67(-11.26,22.61)$ \\
\hline \multicolumn{5}{|c|}{ Pain WOMAC score change } \\
\hline Glu vs. Pla & 10 & 66.3 & 1,069 vs. 1,056 & $-0.75(-1.18,-0.32)^{*}$ \\
\hline Glu vs. NSAIDs & 2 & 85.5 & 425 vs. 423 & $-0.07(-1.5,1.36)$ \\
\hline \multicolumn{5}{|l|}{ Actual score } \\
\hline Glu vs. Pla & 4 & 81 & 408 vs. 418 & $0.06(-1.33,1.45)$ \\
\hline \multicolumn{5}{|c|}{ Functional WOMAC score change } \\
\hline Glu vs. Pla & 10 & 67.6 & 1,069 vs. 1,056 & $-0.58(-1.98,0.81)$ \\
\hline Glu vs. NSAIDs & 2 & 77.1 & 425 vs. 423 & $-0.84(-2.95,4.63)$ \\
\hline \multicolumn{5}{|l|}{ Actual score } \\
\hline Glu vs. Pla & 4 & 90.5 & 408 vs. 418 & $-4.78(-5.96,-3.59)^{*}$ \\
\hline Dia vs. Pla & 2 & 0 & 110 vs. 110 & $-7.72(-18.83,3.39)$ \\
\hline Dia vs. NSAIDs & 2 & 92.9 & 189 vs. 185 & $-6.64(-10.50,-2.78)^{*}$ \\
\hline \multicolumn{5}{|c|}{ Stiffness WOMAC score change } \\
\hline Glu vs. Pla & 7 & 68.1 & 759 vs. 743 & $-0.02(-0.06,0.03)$ \\
\hline \multicolumn{5}{|l|}{ Actual score } \\
\hline Glu vs. Pla & 3 & 31.5 & 390 vs. 389 & $0.09(-0.38,0.56)$ \\
\hline Dia vs. Pla & 2 & 1 & 110 vs. 110 & $-0.68(-1.20,-0.16)^{*}$ \\
\hline \multicolumn{5}{|c|}{ Lequesne score scores change } \\
\hline Glu vs. Pla & 2 & 87.7 & 207 vs. 205 & $-1.03(-1.34,-0.72)^{*}$ \\
\hline Dia vs. Pla & 2 & 0 & 340 vs. 337 & $0.002(-0.704,0.708)$ \\
\hline \multicolumn{5}{|l|}{ Joint space width } \\
\hline Glu vs. Pla & 4 & 86.4 & 357 vs. 350 & $0.008(-0.232,0.248)$ \\
\hline All adverse events & & & & $\operatorname{RR}(95 \% \mathrm{Cl})$ \\
\hline Glu vs. Pla & 16 & 7.3 & 1,366 vs. 1,365 & $1.12(1.02,1.23)^{*}$ \\
\hline Glu vs. NSAIDs & 5 & 81.9 & 631 vs. 632 & $0.53(0.24,1.20)$ \\
\hline Dia vs. Pla & 4 & 76.6 & 275 vs. 278 & $5.58(2.14,14.59)^{*}$ \\
\hline Dia vs. NSAIDs & 2 & 97.6 & 181 vs. 182 & $1.59(0.47,5.44)$ \\
\hline \multicolumn{5}{|l|}{ Gl adverse event } \\
\hline Glu vs. Pla & 14 & 0 & 1,217 vs. 1,211 & $0.99(0.82,1.19)$ \\
\hline Glu vs. NSAIDs & 5 & 74.3 & 631 vs. 632 & $0.51(0.22,1.20)$ \\
\hline Dia vs. Pla & 6 & 94.9 & 937 vs. 701 & $2.00(0.69,5.74)$ \\
\hline Dia vs. NSAIDs & 3 & 86.6 & 373 vs. 267 & $1.37(0.89,2.10)$ \\
\hline
\end{tabular}

*Statistically significant difference $(P<0.05) . \mathrm{Cl}=$ confidence interval, NSAIDs = non-steroidal anti-inflammatory drugs, RR $=$ relative risk, UMD $=$ unstandardized mean difference, $\mathrm{VAS}=$ visual analog score, $\mathrm{WOMAC}=$ Western Ontario and McMaster Universities Osteoarthritis index. 
However, glucosamine did not result in a significant change in joint space when compared to placebo with an UMD of 0.008 (95\% CI: $-0.232,0.248$ ).

\section{Adverse events}

Compared with a placebo control, composite adverse events were 1.12 (95\% CI: 1.02, 1.23) and 5.58 (95\% CI: $2.14,14.59)$ times significantly higher in glucosamine and diacerein than in placebo (Table 2). When considering only GI adverse events, the pooled RR of glucosamine was 0.99 (95\% CI: 0.82, 1.19) when compared with placebo and 0.393 (95\% CI: 0.157, 0.588) when compared with NSAIDs. Conversely, diacerein respectively had 2.00 (95\% CI: 0.69, 5.74) and 1.37 (95\% CI: 0.89, 2.10) times more GI effects than placebo and NSAIDs, but this data was not statistically significant.

\section{Network meta-analysis \\ Visual analog score}

Data from eight studies [39,34,40,42,21,23,25,2] were included in pooling of indirect comparisons of the VAS scores (Additional file 4). Mean VAS scores measured at 4 to 24 weeks after receiving treatments were fitted as the dependent variable in a mixed linear regression model.

The VAS score was lowest in the diacerein group with an overall mean of 3.28 (95\% CI: $2.25,4.30)$ followed by the glucosamine $(3.30,95 \%$ CI: 2.61, 4.01), NSAIDs (3.31, 95\% CI: $2.13,4.50)$, and placebo groups (5.05, 95\% CI: $3.79,6.32)$. The regression analysis suggested that all active treatments resulted in a significant difference in VAS score when compared to placebo (Table 3). Multiple comparisons suggested no difference in effects between active treatments (Figure 2).

\section{Total WOMAC score}

Data from four studies [31,29,20,22] were included in pooling of indirect comparisons of the actual total WOMAC scores (Additional file 4). The mean total WOMAC scores were lower in the diacerein and NSAID groups, but higher in glucosamine compared to placebo, but this was not statistically significant (Table 3). Multiple comparisons suggested no difference in effects between active treatments.

\section{Pain WOMAC score}

Data from six studies [31,28,29,20,22,4] were included in the network meta-analysis of pain WOMAC scores (Additional file 4). Fitting the regression analysis using placebo as the reference suggested that pain WOMAC scores were lower in both the diacerein and NSAIDs groups. In contrast, the pain score was higher in the glucosamine group compared with placebo, but this was not statistically significant (Table 3).
Table 3 Comparisons of treatment effects: a network meta-analysis

\begin{tabular}{|c|c|c|c|c|}
\hline Treatment & $N$ & $B$ & $P$ value & $95 \% \mathrm{Cl}$ \\
\hline \multicolumn{5}{|l|}{ Pain VAS score } \\
\hline Glucosamine & 195 & -1.75 & $0.034^{*}$ & $-3.32,-0.17$ \\
\hline NSAIDs & 292 & -1.74 & $0.047^{*}$ & $-3.44,-0.03$ \\
\hline Diacerein & 209 & -1.78 & $0.024^{*}$ & $-3.24,-0.32$ \\
\hline Placebo & 187 & 0 & - & - \\
\hline Diacerein vs. glucosamine & - & -0.03 & 0.964 & $-1.58,1.52$ \\
\hline NSAIDs vs. glucosamine & - & 0.01 & 0.988 & $-1.52,1.54$ \\
\hline Diacerein vs. NSAIDs & - & -0.04 & 0.893 & $-0.72,0.64$ \\
\hline \multicolumn{5}{|l|}{ Total WOMAC score } \\
\hline Glucosamine & 73 & 17.70 & 0.367 & $-35.46,70.87$ \\
\hline NSAIDs & 79 & -7.03 & 0.334 & $-26.51,12.45$ \\
\hline Diacerein & 164 & -6.2 & 0.597 & $-39.69,27.29$ \\
\hline Placebo & 159 & 0 & - & - \\
\hline Diacerein vs. glucosamine & - & -23.90 & 0.218 & $-72.85,25.06$ \\
\hline NSAIDs vs. glucosamine & - & -24.74 & 0.180 & $-69.91,20.44$ \\
\hline Diacerein vs. NSAIDs & - & 0.84 & 0.899 & $-18.52,20.20$ \\
\hline \multicolumn{5}{|l|}{ Pain WOMAC score } \\
\hline Glucosamine & 408 & 1.47 & 0.139 & $-0.74,3.68$ \\
\hline NSAIDs & 164 & -0.38 & 0.612 & $-2.31,1.55$ \\
\hline Diacerein & 397 & -0.75 & 0.521 & $-3.75,2.24$ \\
\hline Placebo & 491 & 0 & - & - \\
\hline Diacerein vs. glucosamine & - & -2.22 & 0.078 & $-4.84,0.39$ \\
\hline NSAIDs vs. glucosamine & - & -1.85 & 0.065 & $-3.88,0.18$ \\
\hline Diacerein vs. NSAIDs & - & -0.376 & 0.643 & $-2.46,1.71$ \\
\hline \multicolumn{5}{|l|}{ Function WOMAC score } \\
\hline Glucosamine & 408 & -2.42 & 0.534 & $-11.18,6.33$ \\
\hline NSAIDs & 503 & 1.53 & 0.463 & $-3.13,6.20$ \\
\hline Diacerein & 189 & 1.89 & 0.392 & $-3.02,6.81$ \\
\hline Placebo & 407 & 0 & - & - \\
\hline Diacerein vs. glucosamine & - & 4.32 & 0.436 & $-8.03,16.67$ \\
\hline NSAIDs vs. glucosamine & - & 3.96 & 0.438 & $-7.43,15.34$ \\
\hline Diacerein vs. NSAIDs & - & 0.36 & 0.913 & $-7.16,7.89$ \\
\hline \multicolumn{5}{|l|}{ Stiffness WOMAC score } \\
\hline Glucosamine & 390 & -0.15 & 0.480 & $-0.66,0.36$ \\
\hline NSAIDs & 397 & -0.77 & 0.207 & $-2.14,0.60$ \\
\hline Diacerein & 196 & -0.89 & 0.097 & $-2.01,0.23$ \\
\hline Placebo & 499 & 0 & - & - \\
\hline Diacerein vs. glucosamine & - & -0.74 & 0.193 & $2.00,-0.52$ \\
\hline NSAIDs vs. glucosamine & - & 0.62 & 0.205 & $-1.71,0.47$ \\
\hline Diacerein vs. NSAIDs & - & -0.12 & 0.788 & $-1.20,0.96$ \\
\hline \multicolumn{5}{|l|}{ Lequesne score change } \\
\hline Glucosamine & 214 & -1.12 & 0.063 & $-2.36,0.12$ \\
\hline Diacerein & 75 & 0.03 & 0.970 & $-2.13,2.18$ \\
\hline
\end{tabular}


Table 3 Comparisons of treatment effects: a network meta-analysis (Continued)

\begin{tabular}{|c|c|c|c|c|}
\hline Placebo & 192 & 0 & - & - \\
\hline Diacerein vs. glucosamine & - & 1.15 & 0.331 & $-4.32,2.01$ \\
\hline \multicolumn{5}{|l|}{ Joint space width change } \\
\hline Glucosamine & 357 & -0.08 & 0.363 & $-0.31,0.14$ \\
\hline Diacerein & 246 & 0.12 & 0.207 & $-0.10,0.34$ \\
\hline Placebo & 597 & 0 & - & - \\
\hline Diacerein vs. glucosamine & - & -0.20 & $0.001^{*}$ & $-0.27,-0.14$ \\
\hline Adverse event & N & $\mathrm{RR}$ & $P$ value & $95 \% \mathrm{Cl}$ \\
\hline Glucosamine & 1,474 & 1.07 & 0.398 & $0.92,1.23$ \\
\hline NSAIDs & 814 & 2.20 & $<0.001^{*}$ & $1.56,3.11$ \\
\hline Diacerein & 381 & 1.91 & $<0.001^{*}$ & $1.36,2.69$ \\
\hline Placebo & 1,634 & 1 & - & - \\
\hline Diacerein vs. glucosamine & - & 1.80 & $0.001^{*}$ & $1.27,2.55$ \\
\hline NSAIDs vs. glucosamine & - & 2.07 & $<0.001^{*}$ & $1.47,2.91$ \\
\hline Diacerein vs. NSAIDs & - & 0.87 & 0.416 & $0.62,1.22$ \\
\hline \multicolumn{5}{|l|}{ Adverse event (Gl) } \\
\hline Glucosamine & 1,850 & 0.84 & 0.103 & $0.68,1.04$ \\
\hline NSAIDs & 899 & 1.33 & $0.038^{*}$ & $1.02,1.77$ \\
\hline Diacerein & 1,345 & 1.44 & $<0.001^{*}$ & $1.24,1.68$ \\
\hline Placebo & 1,917 & 1 & - & - \\
\hline Diacerein vs. glucosamine & - & 1.72 & $<0.001^{*}$ & $1.34,2.22$ \\
\hline NSAIDs vs. glucosamine & - & 1.60 & $0.004^{*}$ & $1.16,2.19$ \\
\hline Diacerein vs. NSAIDs & - & 1.08 & 0.576 & $0.98,1.50$ \\
\hline
\end{tabular}

*Statistically significant difference $(P<0.05)$. $\mathrm{Cl}=$ confidence interval, $\mathrm{Gl}=$ gastrointestinal, NSAIDs = non-steroidal anti-inflammatory drugs, $\mathrm{RR}=$ relative risk, $\mathrm{VAS}=$ visual analog score, $\mathrm{WOMAC}=$ Western Ontario and McMaster Universities Osteoarthritis index.

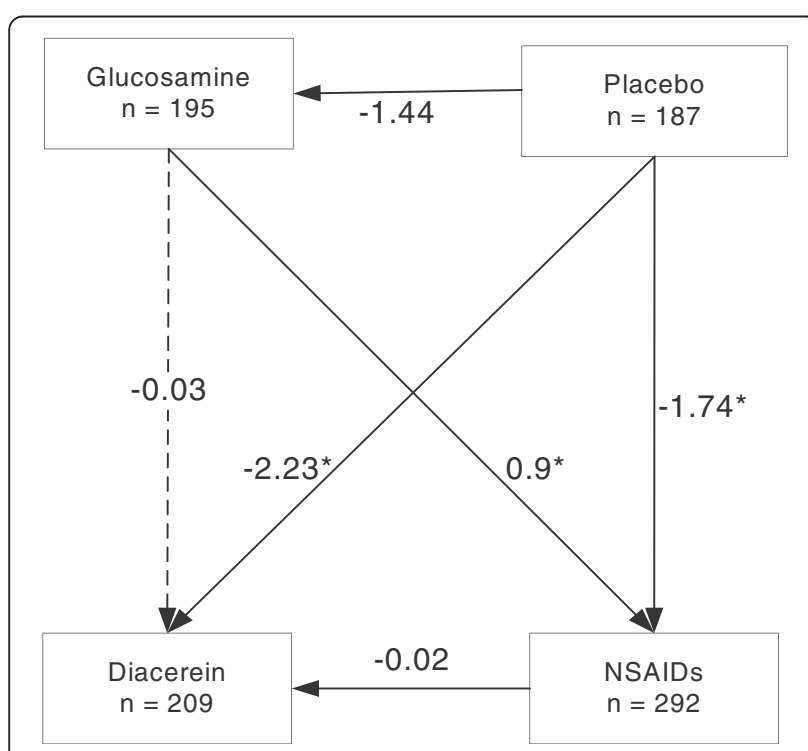

Figure 2 Network meta-analysis of treatment effect on VAS.

\section{Function WOMAC score}

Data from eight studies [20,22,18,31,28,29,4,25] were included in pooling of indirect comparisons of WOMAC function scores (Additional file 4). The regression analysis suggested that mean WOMAC function scores of diacerein, NSAIDs, and glucosamine were lower than placebo, but these results were not statistically significant (Table 3). Multiple comparisons indicated that diacerein and NSAIDs resulted in lower scores than glucosamine, but these results also were not statistically significant.

\section{Stiffness WOMAC score}

Data from six studies [20,22,18,31,28,29] were included in pooling of indirect comparisons of the WOMAC stiffness scores (Additional file 4). The regression analysis suggested that mean WOMAC stiffness scores were lower in diacerein, NSAIDs, and glucosamine groups when compared to placebo (Table 3). There was no significant difference between the three active treatments.

\section{Lequesne algofunctional score change}

Three studies [21,36,37] compared mean changes of Lequesne scores after receiving treatments at 4 to 24 weeks (Additional file 4). The regression analysis suggested that mean Lequesne change in the glucosamine group was lower than the placebo group. There was no significant difference between the glucosamine and diacerein groups.

\section{Joint space width difference}

Data from five studies [33,38,43,41,19] were used for the network meta-analysis of joint space width change. Change of joint space width after receiving glucosamine and diacerein had no statistically significant difference when compared to placebo (Table 3). Multiple comparisons indicated that diacerein was superior to glucosamine at $-0.2 \mathrm{~mm}(95 \% \mathrm{CI}:-0.27,-0.14)$.

\section{Adverse events}

Sixteen studies [39,37,31,42,41,32,38,26,27,35,28,30,29 ,43,47,48] reported overall adverse events between treatment groups (Additional file 5). Compared to glucosamine, NSAIDs was 2.07 (95\% CI: 1.47, 2.91) times and diacerein was 1.80 (95\% CI: $1.27,2.55)$ times more likely to have adverse events (Figure 3). Diacerein had approximately $13 \%(\mathrm{RR}=0.87 ; 95 \% \mathrm{CI}: 0.62,1.22)$ lower risk than NSAIDs, but this was not statistically significant (Table 3). Considering only GI adverse events showed similar results to overall adverse events.

\section{Discussion}

This review compared effects of glucosamine, diacerein, NSAIDs, and placebo for the treatment of osteoarthritis of the knee. Relevant clinical outcomes that were pooled 


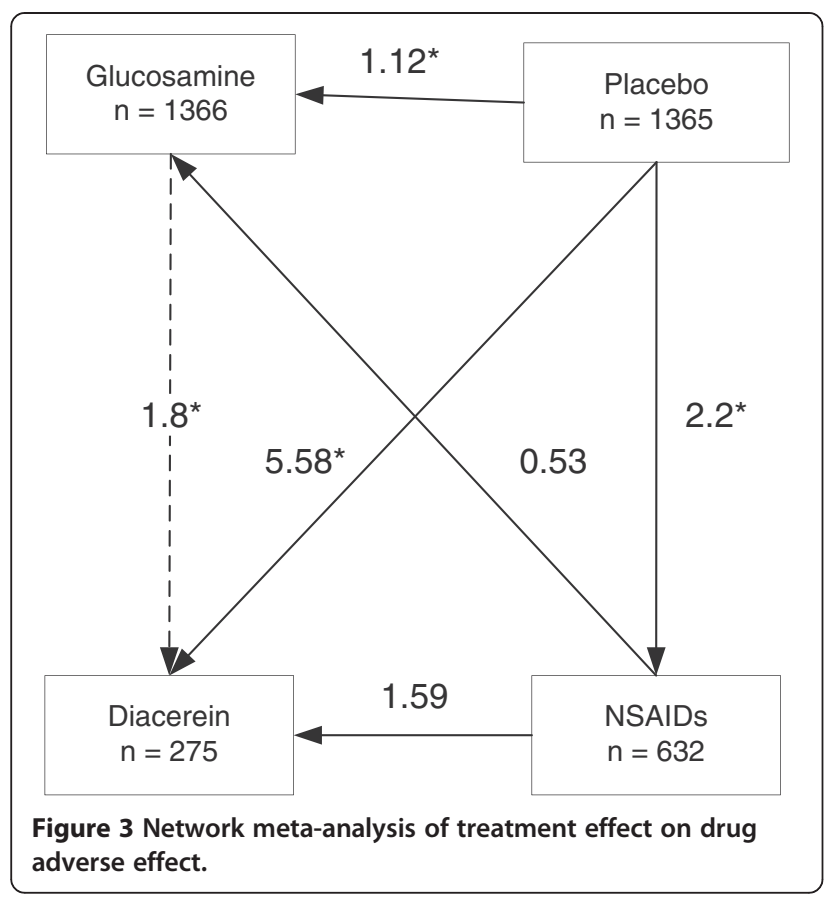

included VAS pain score, total and sub-WOMAC scores, joint space width, and adverse events.

The second-line drugs for OA knee in the SYSADOA group include glucosamine sulfate, glucosamine hydrochloride, chondroitin sulfate, hyaluronic acid, ASU, and diacerein. This study included both glucosamine sulfate and glucosamine hydrochloride because the results of previous meta-analysis of both preparations show that they have no statistically significant difference [49]. Chondroitin sulfate [50-52], hyaluronic acid [53], and ASU [54] were not included in this review because there were too few studies to pooled outcomes with network meta-analysis.

The clinical results of our study were consistent to previous meta-analyses $[6,4,5,3]$ in which glucosamine and diacerein statistically improved pain scores (VAS and WOMAC) and function scores (WOMAC) when compared to placebo. However, we have added more evidence of multiple active treatment comparisons. There were no statistically significant differences between the three groups in pain VAS, total WOMAC, sub-WOMAC scores, and Lequesne functional scores. Although glucosamine showed greater improvement in joint space width when compared to diacerein, glucosamine and diacerein did not show a clinically relevant effect in joint space narrowing when compared to placebo. Both glucosamine and diacerein increased risk of adverse events when compared with placebo. However, glucosamine had a lower risk of adverse events when compared to diacerein. In the subgroup of gastrointestinal adverse events, patients who took diacerein had an approximately $86.9 \%$ and $99.6 \%$ increased risk of GI adverse events when compared to glucosamine and placebo, respectively. Glucosamine and diacerein can reduce pain VAS and improve function (WOMAC). Glucosamine and diacerein showed no differences in adverse effects when compared to NSAIDs. In conclusion, this study demonstrated that either glucosamine or diacerein can be selected for the treatment of pain associated with knee osteoarthritis. Diacerein has a higher risk of adverse GI events when compared to glucosamine. Both glucosamine and diacerein cannot decrease risk of adverse effects, and they both do not have a clinically relevant effect in delaying progression of joint space narrowing in OA of the knee.

The small number of studies that evaluated each particular pair of treatments limits performing a direct meta-analysis. A network meta-analysis circumvents this problem by creating indirect comparisons between active treatments that can identify the most effective therapy. In this case, diacerein was the best therapy for improvement of pain VAS scores. Glucosamine is the best therapy in terms of having less adverse effects when compared to diacerein but not when compared to placebo controls. None of the RCTs had compared combined treatments with an active control.

The strengths of this study were that a network metaanalysis was applied to increase the power of the tests and reduce type I errors [14-16]. We applied a regression model taking into account study effects to assess treatment effects. The network meta-analysis "borrows" treatment information from other studies and increases the total sample size. As a result, treatment effects that could not be detected in direct meta-analysis could be identified. All possible treatment comparisons are mapped and displayed (Additional file 6). Although our pooled estimates were heterogeneous, the regression model with cluster effect takes into account variations at the study level.

None of RCTs compared dual therapy with monotherapy of SYSADOA. In relation to the SYSADOA mechanism, diacerein inhibits IL-1b effects and reduces synthesis of cartilage-specific macromolecules. In addition, diacerein also decreases IL-1b-stimulated secretion of metalloproteinases and aggrecanases, thereby preventing breakdown of cartilage by these enzymes [55]. Glucosamine, an amino sugar, is a building block of the glycosaminoglycan, which is a part of the cartilage structure. The SYSADOA group should be able to support effects of each other and may yield better clinical improvement than monotherapy. Further RCTs that compare dual $v s$. monotherapy SYSADOA are necessary to determine if this may enhance treatment effects.

\section{Conclusions}

This investigation demonstrates the potency of diacerein and glucosamine in the treatment of osteoarthritis of the 
knee. Glucosamine shows significant improvements in pain score but does not decrease risk of adverse effects and does not have a clinically relevant effect in slowing progression of joint space narrowing in OA knee. Diacerein has a higher risk of adverse GI events when compared to glucosamine. Diacerein also does not decrease risk of adverse effects and has no clinically relevant effect in delaying progression of joint space narrowing in $\mathrm{OA}$ of the knee. When compared to diacerein, glucosamine is the better treatment choice for OA of the knee.

\section{Additional files}

\section{Additional file 1: Search Strategies. \\ Additional file 2: Table S2. Risk of bias assessment.}

Additional file 3: Table S3. Direct comparison of means VAS, WOMAC (total, pain, stiffness, and function), Lequesne actual, and difference score according to treatment.

Additional file 4: Table S4. Sample size, mean, and SD between treatment groups for studies included in a network meta-analysis.

Additional file 5: Table S5. Frequency of overall adverse events between treatment groups.

Additional file 6: Table S6. Summarization all treatment effects for osteoarthritis patients.

\section{Abbreviations}

ASU: avocado soybean unsaponifiables; BMI: body mass index; Cl: confidence intervals; Gl: gastrointestinal; NSAIDs: non-steroidal anti-inflammatory drugs; OA: osteoarthritis; RCTs: randomized controlled trials; RR: relative risk; SD: standard deviation; SYSADOA: symptomatic slow-acting drugs for OA; UMD: unstandardized mean difference; VAS: visual analog scores; WOMAC: Western Ontario and McMaster Universities Osteoarthritis index.

\section{Competing interests}

The authors declare that they have no competing interests.

\section{Authors' contributions}

JK contributed to the conception and design, analysis and the interpretation of the data, the drafting of the article, the critical revision of the article for important intellectual content, the final approval of the article, and the collection and assembly of data. TA contributed to the collection and assembly of the data. MM contributed to the conception and design, the drafting of the article, the critical revision of the article for important intellectual content, and the final approval of the article. JA contributed to the conception and design, the drafting of the article, the critical revision of the article for important intellectual content, and the final approval of the article. PW contributed to the conception and design, the drafting of the article, the critical revision of the article for important intellectual content, and the final approval of the article. AT contributed to the conception and design, the analysis and interpretation of the data, the drafting of the article, the critical revision of the article for important intellectual content, the final approval of the article, the statistical expertise, and the collection and assembly of the data. All authors read and approved the final manuscript.

\section{Acknowledgements}

All authors declare that no funding source or any sponsor was involved in the study design, collection, analysis, and interpretation of the data; in the writing of the manuscript; and in the submission to submit the manuscript for publication. This study is a part of Dr.Jatupon Kongtharvonskul 's training in Ph.D. program for Clinical Epidemiology, Faculty of Medicine Ramathibodi Hospital and Faculty of Graduate Studies, Mahidol University, Bangkok, Thailand.

\section{Author details}

${ }^{1}$ Section for Clinical Epidemiology and Biostatistics, Faculty of Medicine, Faculty of Medicine Ramathibodi Hospital, Ratchathewi, Chang Wat Bangkok,
Bangkok 10400, Thailand. ${ }^{2}$ Centre for Clinical Epidemiology and Biostatistics, The University of Newcastle, University Drive, Newcastle NSW 2308, Australia. ${ }^{3}$ Centre for Clinical Epidemiology and Biostatistics, School of Medicine and Public Health, Hunter Medical Research Institute, University of Newcastle, University Drive, Newcastle NSW 2308, Australia. ${ }^{4}$ Department of Orthopaedics, Faculty of Medicine Ramathibodi Hospital, Bangkok, Mahidol University, Highway 3310, Bangkok 73170, Thailand.

Received: 12 September 2014 Accepted: 23 February 2015

Published online: 13 March 2015

\section{Reference}

1. Blandizzi C, Tuccori M, Colucci R, Fornai M, Antonioli L, Ghisu N, et al. Role of coxibs in the strategies for gastrointestinal protection in patients requiring chronic non-steroidal anti-inflammatory therapy. Pharmacol Res Off J Italian Pharmacol Soc. 2009;59(2):90-100. doi:10.1016/j.phrs.2008.11.004.

2. Mukherjee D, Nissen SE, Topol EJ. Risk of cardiovascular events associated with selective COX-2 inhibitors. JAMA. 2001:286(8):954-9.

3. Towheed TE, Maxwell L, Anastassiades TP, Shea B, Houpt J, Robinson V, et al. Glucosamine therapy for treating osteoarthritis. Cochrane Database Syst Rev. 2005;2:CD002946. doi:10.1002/14651858.CD002946.pub2.

4. Rintelen B, Neumann K, Leeb BF. A meta-analysis of controlled clinical studies with diacerein in the treatment of osteoarthritis. Arch Intern Med. 2006;166(17):1899-906. doi:10.1001/archinte.166.17.1899.

5. Wandel $S$, Juni $P$, Tendal B, Nuesch E, Villiger PM, Welton NJ, et al. Effects of glucosamine, chondroitin, or placebo in patients with osteoarthritis of hip or knee: network meta-analysis. BMJ. 2010;341:c4675. doi:10.1136/bmj.c4675.

6. Bartels EM, Bliddal H, Schondorff PK, Altman RD, Zhang W, Christensen R. Symptomatic efficacy and safety of diacerein in the treatment of osteoarthritis: a meta-analysis of randomized placebo-controlled trials. Osteoarthritis Cartilage. 2010;18(3):289-96. doi:10.1016/j.joca.2009.10.006.

7. Liberati A, Altman DG, Tetzlaff J, Mulrow C, Gotzsche PC, loannidis JP, et al. The PRISMA statement for reporting systematic reviews and meta-analyses of studies that evaluate health care interventions: explanation and elaboration. PLoS Med. 2009;6(7):e1000100. doi:10.1371/journal.pmed.1000100.

8. Bellamy N, Buchanan WW, Goldsmith CH, Campbell J, Stitt LW. Validation study of WOMAC: a health status instrument for measuring clinically important patient relevant outcomes to antirheumatic drug therapy in patients with osteoarthritis of the hip or knee. J Rheumatol. 1988;15(12):1833-40.

9. Lequesne MG. The algofunctional indices for hip and knee osteoarthritis. J Rheumatol. 1997:24(4):779-81.

10. Altman DG, Bland JM. Interaction revisited: the difference between two estimates. BMJ. 2003:326(7382):219.

11. Palmer TMPJ, Sutton AJ, Moreno SG. Contour-enhanced funnel plots in meta-analysis. STATA J. 2009;8(2):242-54

12. Peters JL, Sutton AJ, Jones DR, Abrams KR, Rushton L. Contour-enhanced meta-analysis funnel plots help distinguish publication bias from other causes of asymmetry. J Clin Epidemiol. 2008;61(10):991-6. doi:S0895-4356 (07)00435-0.

13. Egger M, Davey Smith G, Schneider M, Minder C. Bias in meta-analysis detected by a simple, graphical test. BMJ. 1997;315(7109):629-34.

14. Song F, Altman DG, Glenny AM, Deeks JJ. Validity of indirect comparison for estimating efficacy of competing interventions: empirical evidence from published meta-analyses. BMJ. 2003;326(7387):472. doi:10.1136/ bmj.326.7387.472.

15. Lu G, Ades AE. Combination of direct and indirect evidence in mixed treatment comparisons. Stat Med. 2004;23(20):3105-24. doi:10.1002/sim.1875.

16. Song F, Harvey I, Lilford R. Adjusted indirect comparison may be less biased than direct comparison for evaluating new pharmaceutical interventions J Clin Epidemiol. 2008;61(5):455-63. doi:10.1016/j.jclinepi.2007.06.006

17. Pilotto A, Franceschi M, Vitale DF, Zaninelli A, Masotti G, Rengo F. Upper gastrointestinal symptoms and therapies in elderly out-patients, users of non-selective NSAIDs or coxibs. Aliment Pharmacol Ther. 2005;22(2):147-55.

18. Brahmachari B, Chatterjee $S$, Ghosh A. Efficacy and safety of diacerein in early knee osteoarthritis: a randomized placebo-controlled trial. Clin Rheumatol. 2009;28(10):1193-8. doi:10.1007/s10067-009-1225-9.

19. Dougados $M$, Nguyen $M$, Berdah L, Mazieres B, Vignon E, Lequesne $M$ Evaluation of the structure-modifying effects of diacerein in hip osteoarthritis: ECHODIAH, a three-year, placebo-controlled trial. Evaluation Chondromodulating Effect Diacerein OA Hip. Arthritis Rheum. 2001;44(11):2539-47. 
20. Louthrenoo W, Nilganuwong S, Aksaranugraha S, Asavatanabodee P, Saengnipanthkul S. The efficacy, safety and carry-over effect of diacerein in the treatment of painful knee osteoarthritis: a randomised, double-blind. NSAID-Controlled Stuty. Osteoarthritis Cartilage. 2007;15(6):605-14 doi:10.1016/j.joca.2007.02.021

21. Nguyen $M$, Dougados $M$, Berdah $L$, Amor B. Diacerein in the treatment of osteoarthritis of the hip. Arthritis Rheum. 1994;37(4):529-36.

22. Pavelka K, Trc T, Karpas K, Vitek P, Sedlackova M, Vlasakova V, et al. The efficacy and safety of diacerein in the treatment of painful osteoarthritis of the knee: a randomized, multicenter, double-blind, placebo-controlled study with primary end points at two months after the end of a three-month treatment period. Arthritis Rheum. 2007:56(12):4055-64. doi:10.1002/art.23056.

23. Pelletier JP, Yaron M, Haraoui B, Cohen P, Nahir MA, Choquette D, etal. Efficacy and safety of diacerein in osteoarthritis of the knee: a double-blind, placebo-controlled trial. The Diacerein Study Group. Arthritis Rheum. 2000;43 (10):2339-48. doi:10.1002/1529-0131(200010)43:10<2339::aid-anr23 > 3.0. co;2-p.

24. Pham T, Le Henanff A, Ravaud P, Dieppe P, Paolozzi L, Dougados M. Evaluation of the symptomatic and structural efficacy of a new hyaluronic acid compound, NRD101, in comparison with diacerein and placebo in a 1 year randomised controlled study in symptomatic knee osteoarthritis. Ann Rheum Dis. 2004;63(12):1611-7. doi:10.1136/ard.2003.019703.

25. Zheng WJ, Tang FL, Li J, Zhang FC, Li ZG, Su Y, et al. Evaluation of efficacy and safety of diacerein in knee osteoarthritis in Chinese patients. Chin Med Sci J. 2006;21(2):75-80

26. Braham R, Dawson B, Goodman C. The effect of glucosamine supplementation on people experiencing regular knee pain. $\mathrm{Br} J$ Sports Med. 2003:37(1):45-9. discussion 9.

27. Cibere J, Kopec JA, Thorne A, Singer J, Canvin J, Robinson DB, et al. Randomized, double-blind, placebo-controlled glucosamine discontinuation trial in knee osteoarthritis. Arthritis Rheum. 2004;51(5):738-45. doi:10.1002/art.20697.

28. Clegg DO, Reda DJ, Harris CL, Klein MA, O'Dell JR, Hooper MM, et al. Glucosamine, chondroitin sulfate, and the two in combination for painful knee osteoarthritis. N Engl J Med. 2006;354(8):795-808. doi:10.1056/ NEJMoa052771.

29. Frestedt JL, Walsh M, Kuskowski MA, Zenk JL. A natural mineral supplement provides relief from knee osteoarthritis symptoms: a randomized controlled pilot trial. Nutr J. 2008;7:9. doi:10.1186/1475-2891-7-9.

30. Herrero-Beaumont G, Ivorra JA, Del Carmen TM, Blanco FJ, Benito P, Martin-Mola E, et al. Glucosamine sulfate in the treatment of knee osteoarthritis symptoms: a randomized, double-blind, placebo-controlled study using acetaminophen as a side comparator. Arthritis Rheum. 2007;56 (2):555-67. doi:10.1002/art.22371.

31. Houpt JB, McMillan R, Wein C, Paget-Dellio SD. Effect of glucosamine hydrochloride in the treatment of pain of osteoarthritis of the knee. J Rheumatol. 1999;26(11):2423-30.

32. Hughes R, Carr A. A randomized, double-blind, placebo-controlled trial of glucosamine sulphate as an analgesic in osteoarthritis of the knee. Rheumatology (Oxford). 2002:41(3):279-84.

33. Kawasaki T, Kurosawa H, Ikeda H, Kim SG, Osawa A, Takazawa Y, et al. Additive effects of glucosamine or risedronate for the treatment of osteoarthritis of the knee combined with home exercise: a prospective randomized 18-month trial. J Bone Miner Metab. 2008;26(3):279-87. doi:10.1007/s00774-007-0813-5.

34. Lopes VA. Double-blind clinical evaluation of the relative efficacy of ibuprofen and glucosamine sulphate in the management of osteoarthrosis of the knee in out-patients. Curr Med Res Opin. 1982;8(3):145-9. doi:10.1185/03007998209112375.

35. McAlindon T, Formica M, LaValley M, Lehmer M, Kabbara K. Effectiveness of glucosamine for symptoms of knee osteoarthritis: results from an internet-based randomized double-blind controlled trial. Am J Med. 2004;117(9):643-9. doi:10.1016/j.amjmed.2004.06.023.

36. Muller-Fassbender H, Bach GL, Haase W, Rovati LC, Setnikar I. Glucosamine sulfate compared to ibuprofen in osteoarthritis of the knee. Osteoarthritis Cartilage. 1994;2(1):61-9.

37. Noack W, Fischer M, Forster KK, Rovati LC, Setnikar I. Glucosamine sulfate in osteoarthritis of the knee. Osteoarthritis Cartilage. 1994;2(1):51-9.

38. Pavelka K, Gatterova J, Olejarova M, Machacek S, Giacovelli G, Rovati LC. Glucosamine sulfate use and delay of progression of knee osteoarthritis: a 3-year, randomized, placebo-controlled, double-blind study. Arch Intern Med. 2002;162(18):2113-23.
39. Pujalte JM, Llavore EP, Ylescupidez FR. Double-blind clinical evaluation of oral glucosamine sulphate in the basic treatment of osteoarthrosis. Curr Med Res Opin. 1980;7(2):110-4. doi:10.1185/03007998009112036.

40. Qiu GX, Gao SN, Giacovelli G, Rovati L, Setnikar I. Efficacy and safety of glucosamine sulfate versus ibuprofen in patients with knee osteoarthritis. Arzneimittelforschung. 1998;48(5):469-74.

41. Reginster JY, Deroisy R, Rovati LC, Lee RL, Lejeune E, Bruyere O, et al. Long-term effects of glucosamine sulphate on osteoarthritis progression: a randomised, placebo-controlled clinical trial. Lancet. 2001;357(9252):251-6. doi:10.1016/s0140-6736(00)03610-2.

42. Rindone JP, Hiller D, Collacott E, Nordhaugen N, Arriola G. Randomized, controlled trial of glucosamine for treating osteoarthritis of the knee. West J Med. 2000;172(2):91-4

43. Rozendaal RM, Koes BW, van Osch GJ, Uitterlinden EJ, Garling EH, Willemsen SP, et al. Effect of glucosamine sulfate on hip osteoarthritis: a randomized trial. Ann Intern Med. 2008;148(4):268-77.

44. Sawitzke AD, Shi H, Finco MF, Dunlop DD, Bingham 3rd CO, Harris $C L$, et al. The effect of glucosamine and/or chondroitin sulfate on the progression of knee osteoarthritis: a report from the glucosamine/chondroitin arthritis intervention trial. Arthritis Rheum. 2008;58(10):3183-91. doi:10.1002/art.23973.

45. Durmus D, Alayli G, Bayrak IK, Canturk F. Assessment of the effect of glucosamine sulfate and exercise on knee cartilage using magnetic resonance imaging in patients with knee osteoarthritis: a randomized controlled clinical trial. J Back Musculoskeletal Rehabil. 2012;25(4):275-84 doi:10.3233/bmr-2012-0336

46. Kwoh CK, Roemer FW, Hannon MJ, Moore CE, Jakicic JM, Guermazi A et al. Effect of oral glucosamine on joint structure in individuals with chronic knee pain: a randomized, placebo-controlled clinical trial. Arthritis Rheumatol (Hoboken NJ). 2014;66(4):930-9. doi:10.1002/art.38314.

47. Madhu K, Chanda K, Saji MJ. Safety and efficacy of Curcuma longa extract in the treatment of painful knee osteoarthritis: a randomized placebocontrolled trial. Inflammopharmacology. 2013;21(2):129-36. doi:10.1007/s10787-012-0163-3.

48. Chopra A, Saluja M, Tillu G, Sarmukkaddam S, Venugopalan A, Narsimulu G, et al. Ayurvedic medicine offers a good alternative to glucosamine and celecoxib in the treatment of symptomatic knee osteoarthritis: a randomized, double-blind, controlled equivalence drug trial. Rheumatology (Oxford). 2013;52(8):1408-17. doi:10.1093/rheumatology/kes414.

49. Wu D, Huang Y, Gu Y, Fan W. Efficacies of different preparations of glucosamine for the treatment of osteoarthritis: a meta-analysis of randomised, double-blind, placebo-controlled trials. Int J Clin Pract. 2013:67(6):585-94. doi:10.1111/ijcp.12115.

50. Michel BA, Stucki G, Frey D, De Vathaire F, Vignon E, Bruehlmann P, et al. Chondroitins 4 and 6 sulfate in osteoarthritis of the knee: a randomized, controlled trial. Arthritis Rheum. 2005;52(3):779-86. doi:10.1002/art.20867.

51. Mazieres B, Hucher M, Zaim M, Garnero P. Effect of chondroitin sulphate in symptomatic knee osteoarthritis: a multicentre, randomised, double-blind, placebo-controlled study. Ann Rheum Dis. 2007:66(5):639-45. doi:10.1136/ard.2006.059899.

52. Kahan A, Uebelhart D, De Vathaire F, Delmas PD, Reginster JY. Long-term effects of chondroitins 4 and 6 sulfate on knee osteoarthritis: the study on osteoarthritis progression prevention, a two-year, randomized, double-blind, placebo-controlled trial. Arthritis Rheum. 2009;60(2):524-33. doi:10.1002/art.24255.

53. Tashiro T, Seino S, Sato T, Matsuoka R, Masuda Y, Fukui N. Oral administration of polymer hyaluronic acid alleviates symptoms of knee osteoarthritis: a double-blind, placebo-controlled study over a 12-month period. TheScientificWorldJOURNAL. 2012;2012:167928. doi:10.1100/2012/167928.

54. Appelboom T, Schuermans J, Verbruggen G, Henrotin Y, Reginster JY. Symptoms modifying effect of avocado/soybean unsaponifiables (ASU) in knee osteoarthritis. A double blind, prospective, placebo-controlled study. Scand J Rheumatol. 2001:30(4):242-7.

55. Pujol JP, Chadjichristos C, Legendre F, Bauge C, Beauchef G, Andriamanalijaona $\mathrm{R}$, et al. Interleukin-1 and transforming growth factorbeta 1 as crucial factors in osteoarthritic cartilage metabolism. Connect Tissue Res. 2008:49(3):293-7. doi:10.1080/03008200802148355. 